\title{
Alignment and Calibration of the Belle II Detector
}

\author{
Tadeáš Bilka, ${ }^{1, *}$, Kirill Chilikin ${ }^{2}$, David Dossett ${ }^{3}$, Yinghui Guan ${ }^{4}$, Jakub Kandra ${ }^{1}$, Claus \\ Kleinwort $^{5}$, and Martin Sevior ${ }^{3}$ \\ ${ }^{1}$ Faculty of Mathematics and Physics, Charles University, 12116 Prague, Czech Republic \\ ${ }^{2}$ P.N. Lebedev Physical Institute of the Russian Academy of Sciences, Moscow 119991, Russian Fed- \\ eration \\ ${ }^{3}$ School of Physics, University of Melbourne, Victoria 3010, Australia \\ ${ }^{4}$ Duke University, Durham, North Carolina 27708, U.S.A. \\ ${ }^{5}$ Deutsches Elektronen-Synchrotron, 22607 Hamburg, Germany
}

\begin{abstract}
In spring 2018 the SuperKEKB electron-positron collider at High Energy Accelerator Research Organization (KEK, Tsukuba, Japan) will deliver its first collisions to the Belle II experiment. The aim of Belle II is to collect a data sample 50 times larger than the previous generation of BFactories taking advantage of the unprecedented SuperKEKB design luminosity of $8 \times 10^{35} \mathrm{~cm}^{-2} \mathrm{~s}^{-1}$. The Belle II detector will allow to conduct precise measurements in the harsh collider environment, probing for signs of physics beyond the standard model at the precision frontier.

In order to deliver data suitable for physics analysis, the detector has to be properly calibrated on a regular basis. Among other calibrations the detector alignment plays a key role. For example, precise measurements of time dependent $\mathrm{CP}$-violation rely on the accurate alignment of the new vertex detector, as well as on the determination of the beamspot position and size. To automate the calibration procedures and manage the large amount of data and processing power needed for detector calibration, a software framework has been developed which allows to define the complete workflow and to execute it on a computing cluster. The framework integrates the Millepede II algorithm to solve the large minimization problem emerging in the track-based alignment and calibration of the pixel and strip detector, the central drift chamber, and the muon system.

The first collision data will allow to test and to further improve and tune the alignment and calibration procedures. Although the vertexing capabilities will be limited due to the installation of only a small slice of the full vertex detector, the commissioning phase will allow to test most of the alignment procedure features and to prepare for the full operation. We will present the results achieved during the first data taking, the experience gained and the plans for the first physics run with the full detector.
\end{abstract}

\section{Introduction}

On April 26, 2018 (00:38 JST), the SuperKEKB collider delivered the first collisions for the Belle II experiment [1]. SuperKEKB is a so called $B$-Factory: an asymmetric electronpositron collider with center-of-mass energy of the $\Upsilon(4 S)$ resonance. It is an upgrade of

\footnotetext{
*e-mail: bilka@ipnp.mff.cuni.cz
} 


\section{Belle II Detector}

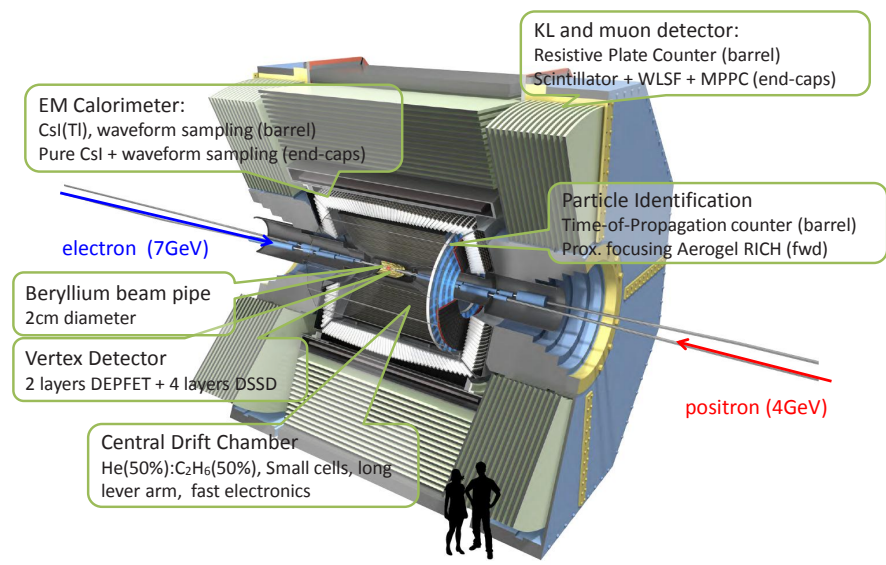

Figure 1. Cross-section of the Belle II detector with its sub-detector systems: vertex detector, drift chamber, particle identification systems, electromagnetic calorimeter and the muon system which also serves as a return yoke for the $1.5 \mathrm{~T}$ superconducting magnet. Main new and/or upgraded features of each sub-detector are highlighted in the captions.

former KEKB accelerator with design instantaneous luminosity of $8 \times 10^{35} \mathrm{~cm}^{-2} \mathrm{~s}^{-1}$ and together with Belle II experiment it aims to collect 50 times larger data sample than the previous generation of $B$-Factories. This will allow to conduct precise measurements in the field of heavy flavour physics and probe physics beyond standard model at the precision frontier [2].

The upgraded accelerator will mean new challenges for the Belle II detector, installed at the interaction point. The detector will have to cope with much higher event rate $(30 \mathrm{kHz})$, detector occupancy, beam-induced backgrounds and radiation damage. Therefore a substantial upgrade of the detector is necessary. One of the key new features will be a completely new vertex detector (VXD). VXD is designed as a six layer silicon detector, with two innermost layers using DEPFET [3] pixel sensors (PXD, [4]) and 4 layers of strip sensors (SVD, [5]). Surrounded by a central drift chamber (CDC), particle identification device and an electromagnetic calorimeter, it is in the center of an $1.5 \mathrm{~T}$ solenoid superconduncting magnet, which construction is also part of the muon and $K_{L}$ detection system (KLM), see Figure 1.

The increased statistics for physics measurements needs to be complemented with improved systematic uncertainties reflected in improved resolution and performance of the individual sub-detectors. Novel techniques are also employed in the experiment software, from track finding and tracking to new methods for alignment and calibration of the detector, which will be crucial to reach the designed performance of Belle II. Namely the vertex detector alignment quality will play a crucial role in precise measurements of time dependent CPviolation parameters, as well as accurate determination of the primary beam spot. Procedures for detector alignment and calibration being developed and exercised on the first data are presented in this article, with some emphasis on the vertex detector and the overall software framework common to other sub-detectors.

\section{Commissioning Phase 2}

The start-up of the Belle II experiment is divided into three phases. Phase 1 was devoted to the commissioning of the SuperKEKB accelerator with orbiting beams but without collisions. In Phase 2, the beams were collided for the first time. The aim was to tune the collision optics and evaluate the various beam backgrounds with dedicated radiation monitoring detectors (so called Beast II sub-detector) and reduced version of the VXD. The ultimate goal of this phase was to ensure safe environment for the full scale vertex detector and to gain experience with 
data acquisition and operation of the individual sub-detector systems. Phase 2 started in April 2018 and on April 26, the first collisions were recorded by the Belle II detector. Until the end of Phase 2 in mid-July 2018, the experiment collected the first $\sim 500 \mathrm{pb}^{-1}$ of collision data. In March 2019, the final setup of the experiment, with full VXD, which is being installed during second half of 2018, should start to collect physics data in so called early Phase 3.

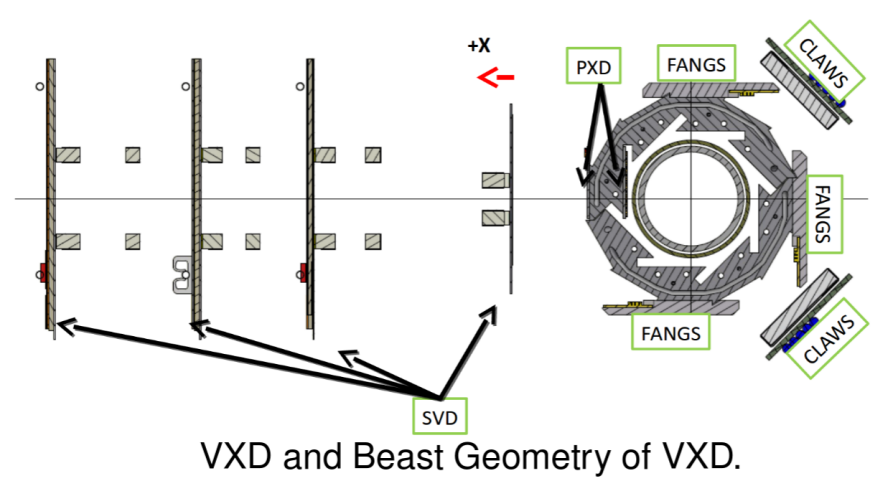

Figure 2. Schematic drawing of the vertex detector in Phase 2. The pixel and strip detector sensors (denoted as PXD and SVD in the figure) are complemented with additional dedicated radiation detectors (known as Beast sub-detector) in the Phase 2 setup. Slanted strip sensors are not shown for clarity.

\subsection{Phase 2 VXD}

The vertexing capabilities of VXD are limited in Phase 2, because only a section of the full VXD is installed. Nevertheless, many aspects of the detector can be already tested during this period, including the calibration and alignment procedures. The reduced VXD for Phase 2 is utilizing the same sensor types as the full setup. It is limited to only single ladder in each layer in horizontal arrangement, see Figure 2. In total there are 22 silicon sensors (4 pixel + 14 strip sensors).

The two innermost layers are placed at radii of $14 \mathrm{~mm}$ and $22 \mathrm{~mm}$ from the beam line and are composed from four $75 \mu \mathrm{m}$ thick DEPFET sensors (two of them in each layer), with pixel size $50 \mu \mathrm{m} \times 55-60 \mu \mathrm{m}$ in first layer and $50 \mu \mathrm{m} \times 70-85 \mu \mathrm{m}$ in the second layer, with smaller pitch in the central region, nearest to the interaction point.

The strip sensors are placed at radii ranging from $38 \mathrm{~mm}$ to $140 \mathrm{~mm}$ and are divided to barrel and forward part, which utilizes slanted sensors with trapezoidal shape to reduce the material budget in forward acceptance region. Barrel and slanted sensors have thickness of $300 \mu \mathrm{m}$ and $320 \mu \mathrm{m}$ respectively. The outer sensors have pitch of $75 \mu \mathrm{m}$ along $z$ and $240 \mu \mathrm{m}$ in $r-\phi$ direction, while the innermost strip layer uses smaller pitch $(50 \mu \mathrm{m}$ along $z$ and 160 $\mu \mathrm{m}$ in $r-\phi$ direction). For the slanted sensors, the pitch size varies along the $z$ coordinate.

\section{Alignment and Calibration Software}

Calibration and alignment is crucial to reach the designed physics performance of the detector. Millions of calibration constants are needed for detector operation, data reconstruction and analysis. Many of the constants can only be determined using the acquired data. Some of the constants are related to individual sub-detector calibration and can be determined by the dedicated sub-detector systems and software, an example is determination of the pedestals in the pixel sensors. We call such calibrations local calibration. These are usually produced by the detector sub-systems regularly before the actual physics data taking, as they are usually independent of other sub-detectors. On top of the local calibrations, there is a large number of constants which are produced promptly after the data taking or offline. The interplay of the 
sub-detectors and reconstruction software is necessary to determine such constants. In Belle II, the aim is to integrate the procedures in the common experiment software framework, called basf2 (Belle II analysis software framework) [6].

Inside basf2, we develop a common Calibration and Alignment Framework (CAF) to produce such constants in an automated way. A lot of efforts is devoted to provide a common ground for such procedures, allowing the developers, who are usually physicists, to concentrate on the actual calibration algorithms. CAF is designed to simplify the development of the calibration procedures, define dependencies among the calibrations, manage possible iterations, data aggregation and reconstruction, job submission on a computing cluster or communication with data database. This is achieved by splitting the calibration into so called collection and algorithm classes. These classes are written in $\mathrm{C}++$ and are re-using many features of basf 2 to perform data and processing intensive tasks. The workflow logic itself, as well as definitions and configuration of the workflow, is written in Python, the scripting language of basf2, which allows more flexible development.

Many of the algorithms for determination of relevant constants for the various subdetectors are already integrated in CAF. We discuss in more detail only a relatively small subset of those, namely those using a common track-based alignment and calibration utilizing the Millepede II tool [7]. Millepede II is a linearized least squares minimization method designed for determination of large number of calibration constants based on minimization of normalized track-to-hit residuals. The parameters influencing the resulting $\chi^{2}$ expression

$$
\chi^{2}\left(\vec{p}, \overrightarrow{q_{1}}, \overrightarrow{q_{2}}, \ldots\right)=\sum_{i}^{\text {tracks }} \sum^{\text {measurements }}\left(\frac{\text { measurement }-\operatorname{prediction}\left(\vec{p}, \vec{q}_{i}\right)}{\text { resolution }}\right)^{2}
$$

are separated into two categories. The global parameters $\vec{p}$ are the calibration parameters of interest. The so called local parameters of the individual tracks used for calibration, denoted as $\overrightarrow{q_{1}}, \overrightarrow{q_{2}}, \ldots$, are also subject to simultaneous minimization, but their values are not determined by Millepede II. Instead, block matrix algebra is used to reduce the problem, which would otherwise lead to a huge matrix equation (with dimension proportional to number of used tracks and their parameters), to a much smaller matrix equation of the dimension equal to the number of global parameters to be determined. Such approach allows to efficiently solve the minimization problem while keeping all the possible correlations in the solution. The problem is solved without any approximation, except linearization. To account for non-linearities, the procedure can be iterated with an updated linearization point. [8]



Figure 3. Definition of the rigid body alignment parameters. Three translation parameters $(u, v, w)$ correspond to shifts in the local reference system of a rigid body. The three rotation angles correspond to small rotations $(\alpha, \beta, \gamma)$ around the respective local axes.

To fully utilize the Millepede II method, the tracks, which are output of standard reconstruction procedures are re-fitted using the General Broken Lines (GBL) method [9, 10] integrated into the GENFIT2 toolkit [11], which is a common track-fitting method used at Belle II. To each measurement on a track, a set of derivatives of the measurement residuals with respect to some calibration or alignment parameters can be added and determined by 
Millepede. So far, four sub-detectors have been integrated into the procedure. For the PXD and SVD, as well as for the KLM, the alignment constants are determined for the individual sensors or modules, treating them as rigid bodies with six alignment parameters, see Figure 3. In addition, for the VXD the positions and orientations of higher level structures (ladders, half-shells) can be used (with proper equality constraints fixing the additional degrees of freedom) as additional alignment parameters. For CDC, alignment of its layers is considered, as well as individual wires, together with additional calibration constants influencing the trackto-hit residual. For example the $T_{0}$ calibration has to be determined for each channel (wire), as well as gravitational wire sagging or parameters of the $x-t$ relations. Many more constants are considered for calibration with Millepede II and are currently under various level of development and testing. In addition, the method also allows to calibrate the position of the primary beamspot if vertex and beam constrained decays are used as input.

\section{Experience and Results with First Data}

Before the start of Phase 2, the Belle II detector was collecting cosmic ray data in the so called global cosmic run (GCR). These data were used for initial alignment and calibration of the detector. For the Phase 2, the situation is quite special - we discuss its alignment with GCR and first collision data in deeper detail here. While the full VXD setup could be in principle aligned using only VXD-only data (fixing the trivial six degrees of freedom corresponding to global shifts and rotation of the sub-detector), for the reduced setup the information from CDC plays a crucial role. The poorly determined degrees of freedom are fixed by considering the alignment and calibration of CDC fixed in the alignment procedure.

The alignment of VXD using GCR data suffers from limited statistics due to relatively small volume occupied by the vertex detector and its horizontal layout, which together with the natural $\phi$ asymmetry of the muon cosmic tracks, means tracks with rather shallow angles are collected (mainly without magnetic field). Also the data sample without magnetic field was quite limited and could not be used for the alignment.

The data with magnetic field collected in GCR were used for the initial alignment and calibration of CDC. Other sub-detectors rely on the CDC tracks for in their calibration procedures. After the first collisions in Phase 2, the full set of calibrations is performed after the data is centrally re-processed. Several iterations are needed to improve the performance, due to dependencies among the individual calibration algorithms. These dependencies will be reflected in the prepared worflows in CAF.

After the CDC calibration and alignment reached sufficient precision, the alignment of the VXD was possible. For the initial alignment, the GCR data were used and the most significant corrections to the sensor position with respect to nominal geometry were estimated. After the first collisions, GCR data were combined with collision data to further improve the alignment. Tracks with VXD hits are re-fitted by GBL and continue to CDC and KLM, which serve as an external reference. All six rigid body parameters for all VXD sensors are free in the alignment procedure, where in total 108 (18 sensors $\times 6$ rigid body parameters) alignment parameters were determined in the simultaneous fit by Millepede II. The improvement in track-to-hit residuals after the alignment with first collisions is clearly demonstrated in Figure 4.

All 3k reconstructed charged tracks with at least four VXD hits accumulated in the first physics run plus about 50k cosmic tracks (with at least one VXD hit) were used for alignment. The achieved alignment was finally checked with higher-level physics analysis and distributions, for example the evaluation of the size of interaction region profile is shown in Figure 5. The VXD alignment is later checked with additional data collected by the experiment for stability in time, showing only small variations without any systematic evolution. 


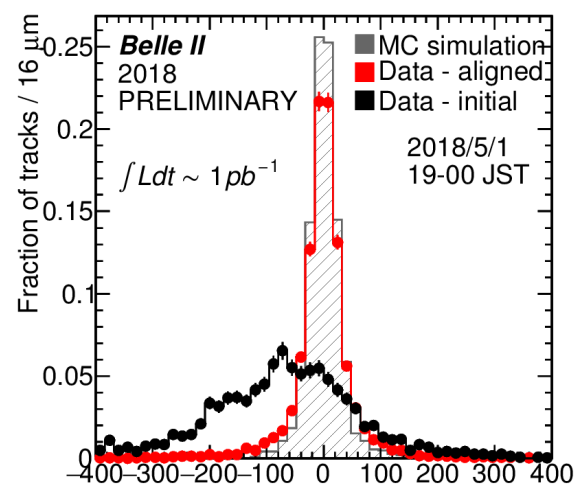

Track-to-hit residual (v-coordinate) $[\mu \mathrm{m}]$

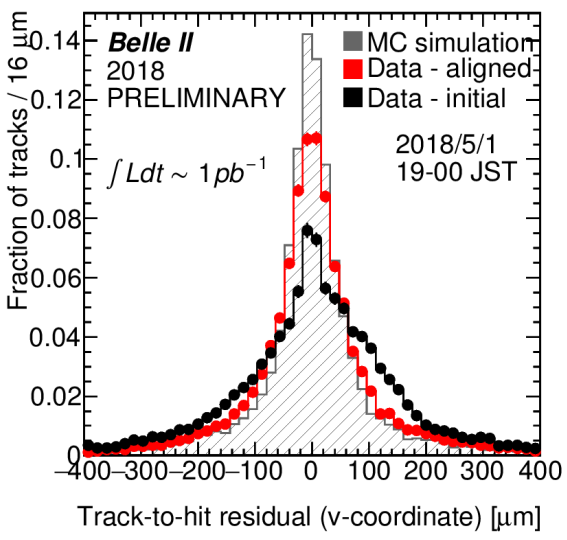

Figure 4. Histograms from alignment DQM modules show track-to-hit residuals from all PXD (left) and SVD (right) sensors after standard reconstruction and track fitting is performed. Distributions are shown for the residuals of the local v-coordinate, which is parallel, neglecting local alignment rotations, to the nominal $\mathrm{z}$ axis, i.e. the Belle II solenoid axis pointing toward the forward part of the detector. The data from some of the first collisions are used to illustrate the improvement of the position resolution before (black) and after (red) the first preliminary alignment is computed and used in reconstruction. A sample of $20 \mathrm{k}$ charged particles originating near IP (electrons, positrons, protons) is generated to check the correspondence of the data and MC simulation (grey). All compared histograms are normalized.

On average the precision of the alignment was estimated by the validation to be better than 10 (20) $\mu m$ for the pixel (strip) sensors for the VXD during the whole Phase 2.

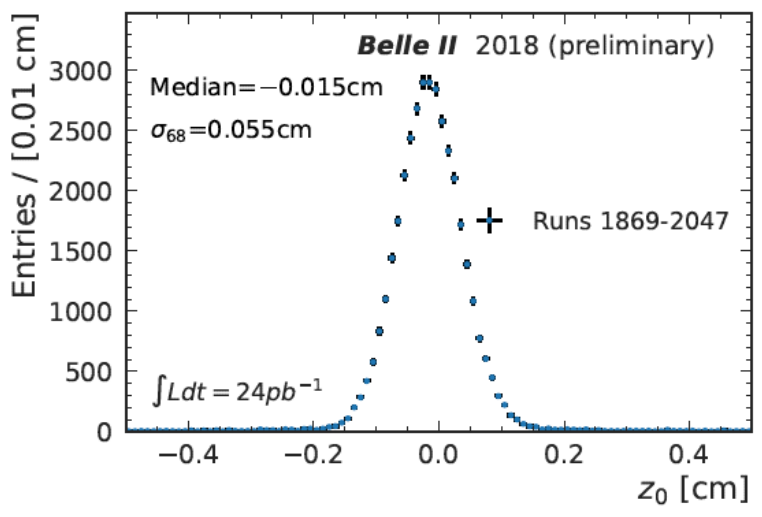

Figure 5. The interaction region profile in the longitudinal (z) direction, measured using the point of origin of charged particle tracks in the Belle II detector, showing the currently achieved interaction region longitudinal size of $0.55 \mathrm{~mm}$.

\section{Conclusions and Plans}

There are many efforts underway in the collaboration on software side towards automation and development of tools for reliable, fast and efficient production of the calibration and alignment constants. One of them is the integration of various sub-detectors and constants in the common global Millepede II calibration procedure. We successfully exercised various procedures and algorithms with cosmic and first collision data. The alignment of VXD is 
showing very good results and was validated also with successive analysis of physics data, but many additional tests are foreseen with the Phase 2 data, namely the alignment and calibration of CDC and KLM with Millepede II or usage of vertex and beam or mass-constrained decays to improve the systematics. Most of the features for pixel, strip, drift chamber and the muon detector alignment and calibration are implemented in the software and should be included in the production of the constants before Phase 3.

\section{Acknowledgments}

The study was supported by the Charles University (project GA UK No. 404316), Ministry of education, youth and sports (Czech Republic) and MSCA-RISE project JENNIFER (EU grant n. 644294).

\section{References}

[1] Z. Doležal, S. Uno et al., Belle II Technical Design Report (KEK, Japan, 2013)

[2] A. Bevan et al., The Physics of the B Factories (Springer, Germany, 2015)

[3] DEPFET Collaboration, IEEE Trans. Nucl. Sci. 60, 1457 - 1465 (2013)

[4] C. Marinas et al., Nucl. Instrum. Meth. A 731, 31 - 35 (2013)

[5] M. Friedl et al., Nucl. Instrum. Meth. A 732, 83 - 86 (2013)

[6] T. Kuhr et al., Comput.Softw.Big Sci. 3 1, 1 - 12 (2019)

[7] Physics at the Terascale, https://www.wiki.terascale.de/index.php/Millepede_II

[8] V. Blobel, C. Kleinwort, F. Meier, Comput. Phys. Commun. 182, 1760 - 1763 (2011)

[9] C. Kleinwort, Nucl.Instrum.Meth. A 673, 107 - 110 (2012)

[10] Physics at the Terascale, https://www.wiki.terascale.de/index.php/GeneralBrokenLines

[11] C. Höppner et al., Nucl.Instrum.Meth. A 620, 518 - 525 (2010) 